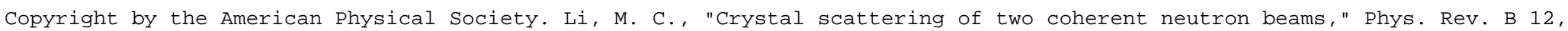
3150 DOI: http://dx.doi.org/10.1103/PhysRevB.12.3150

\title{
Crystal scattering of two coherent neutron beams
}

\author{
Ming Chiang Li \\ Department of Physics, Virginia Polytechnic Institute, Blacksburg, Virginia 24061 \\ and State University, Blacksburg, Virginia 24061
}

(Received 25 February 1975)

\begin{abstract}
The crystal scattering of two coherent neutron beams is discussed. It is found that the conventional pair correlation function used in single-beam scattering is insufficient to describe the scattering of two coherent neutron beams. A generalized pair correlation function with unequal momentum transfer is introduced. It is found that the energy exchange between vibrational quanta of the crystal and incident neutrons leads to a modification of the scattering amplitude. Through the scattering of two coherent neutron beams one is able to measure directly the phase of the scattering amplitude.
\end{abstract}

\section{INTRODUCTION}

The crystal scattering of two coherent beams was carried out experimentally by Marton, Simpson, and Suddeth ${ }^{1}$ more than twenty years ago. In the experiment, two coherent electron beams with energy of $60 \mathrm{keV}$ were used. Their objective was to show directly the interference effect of electron beams. The resulting interference fringes were very distinct, showing strong contrast, and were steady. Unfortunately, the importance of their experiment was overlooked. The data were never analyzed and the experiment has not been repeated since.

It has been realized only recently ${ }^{2}$ that the scattering of two coherent beams could provide more information than that of a conventional single beam. It may actually enable us to determine the phase of the structure factor, which is sometimes called the coherent atomic scattering amplitude and which has not been determined experimentally. Thus, the scattering of two coherent beams affords a new method of experimental research.

In previous discussions, we neglected the displacement of the nuclei from their equilibrium positions, and assumed the lattice to be perfect. In a real crystal, the nuclei are subjected to displacements from their ideal lattice positions, and the scattered amplitude will depend on these displacements. ${ }^{3}$ It is the intention of this paper to study such a dependency.

In conventional scattering experiments, various kinds of beams may be used. Not all of these beams are suitable for initiating scattering of two coherent beams. ${ }^{4}$ The coherent beams are formed through the division of a primary beam. There are stringent requirements on such a beam. First, its source has to be very well defined in energy and position. Second, the path differences intro- duced into the coherent beams must be smaller than the coherence length of the original beam. At the present time, the coherent beams most often used in experimental work are those derived from light, electron, ${ }^{5}$ or neutron ${ }^{6}$ sources .

Coherent neutron beams are beams of thermal neutrons with wavelengths of about $4 \AA$. The cry stal scattering of these beams needs more careful study than that of coherent electron beams. The reason for this is not difficult to explain. Thermal neutrons have energies comparable to that of lattice vibrations and the energy exchanges between them are not negligible. Hence, as the crystal diffraction of two coherent neutron beams is concerned, the present study is important.

For convenience, the incident beams referred to in this paper will be called the neutron beams. The results may be applied to other coherent beams. In Sec. II the general theory for coherent neutron scattering by a system of interacting particles is discussed in the Born approximation. It is found that the conventional pair-correlation function ${ }^{7}$ used in the single-beam scattering is insufficient to describe the scattering of two coherent neutron beams. Therefore, a generalized pair-correlation function is introduced. It depends on the momentum difference between two incident coherent beams. In Sec. III coherent scattering by a harmonically bounded nucleus is studied to determine the effects of temperature on the scattering process. It is found that the coherent differential cross section depends on the temperature of the nucleus. The crystal scattering of two coherent neutron beams is discussed in Sec. IV. It is shown that the energy exchange between the vibrational quanta of the crystal and incident neutrons leads to a modification of the scattering amplitude. Thermal vibrations do not destroy the coherent effect. The phase of the scattering amplitude is still measurable. 


\section{SCATTERING FORMULA}

In the discussion below, it is assumed that the interaction between the incident neutron and the target nuclei is of the general form

$$
H_{\text {int }}=\sum_{j} U\left(\overrightarrow{\mathbf{r}}-\overrightarrow{\mathrm{R}}_{j}\right),
$$

where $\vec{r}$ and $\vec{R}_{j}$ are the position vectors of the incident neutron and the $j$ th target nucleus, respectively. An incident neutron in the two coherent beams can be described by the wave function

$$
\left|\overrightarrow{\mathrm{k}}_{1}\right\rangle+a\left|\overrightarrow{\mathrm{k}}_{2}\right\rangle=\frac{1}{\sqrt{V}} e^{i \overrightarrow{\mathrm{k}}_{1} \cdot \overrightarrow{\mathrm{r}}}+a \frac{1}{\sqrt{V}} e^{i \overrightarrow{\mathrm{k}}_{2} \cdot \overrightarrow{\mathrm{r}}}
$$

where $V$ is the normalization volume. The factor $a$ denotes the relative phase and amplitude of these two beams. To be coherent, these two beams may be different in direction, but must be the same in energy,

$$
E=\hbar^{2} k_{1}^{2} / 2 m=\hbar^{2} k_{2}^{2} / 2 m=\hbar^{2} k^{2} / 2 m .
$$

$m$ is the mass of the incident neutron. According to the Born approximation, the differential cross section for the scattering process in which the incident neutron state $\left|\vec{k}_{1}\right\rangle+a\left|\vec{k}_{2}\right\rangle$ changes to $\left|\vec{k}^{\prime}\right\rangle$, and the state of the target nuclei changes from $|\lambda\rangle$ to $\left|\lambda^{\prime}\right\rangle$ is

$$
\begin{aligned}
\frac{d^{2} \sigma}{d \Omega d E}= & \frac{k^{\prime}}{\left(1+|a|^{2}\right) k}\left(\frac{m}{2 \pi \hbar^{2}}\right)^{2} \mid\left\langle\overrightarrow{\mathrm{k}}^{\prime}, \lambda^{\prime}\left|H_{\mathrm{int}}\right| \overrightarrow{\mathrm{k}}_{1}, \lambda\right\rangle \\
& +\left.a\left\langle\overrightarrow{\mathrm{k}}^{\prime}, \lambda^{\prime}\left|H_{\mathrm{int}}\right| \overrightarrow{\mathrm{k}}_{2}, \lambda\right\rangle\right|^{2} \delta\left(\hbar \omega+E_{\lambda}-E_{\lambda}^{\prime}\right),
\end{aligned}
$$

where

$$
\hbar \omega=\frac{\hbar^{2}}{2 m}\left(k^{2}-k^{\prime 2}\right)=E-E^{\prime} .
$$

The initial and final energies of target nuclei have been denoted as $E_{\lambda}$ and $E_{\lambda^{\prime}}$. In order to obtain the total partial differential cross section for all pos- sible scattering processes, one must sum over all the final states of the target nuclei, and average over all the initial states, which occur with probabilities $p_{\lambda}$; thus,

$$
\begin{aligned}
\frac{d \sigma}{d \Omega d E^{\prime}}= & \frac{k^{\prime}}{\left(1+|a|^{2}\right) k}\left(\frac{m}{2 \pi h^{2}}\right)^{2} \sum_{\lambda, \lambda^{\prime}} P_{\lambda} \\
& \times \mid\left\langle\overrightarrow{\mathrm{k}}^{\prime}, \lambda^{\prime}\left|H_{\mathrm{int}}\right| \overrightarrow{\mathrm{k}}_{1}, \lambda\right\rangle \\
& +\left.a\left\langle k^{\prime}, \lambda^{\prime}\left|H_{\mathrm{int}}\right| k_{2}, \lambda\right\rangle\right|^{2} \delta\left(\hbar w+E_{\lambda}-E_{\lambda^{\prime}}\right) .
\end{aligned}
$$

Substituting Eq. (2.1) into Eq. (2.6), one obtains

$$
\frac{d^{2} \sigma}{d \Omega d E^{\prime}}=\frac{N k^{\prime}}{\left(1+|a|^{2}\right) k}\left(\frac{m}{2 \pi \hbar^{2}}\right)^{2} S\left(\overrightarrow{\mathrm{k}}_{2}-\overrightarrow{\mathrm{k}}_{1} ; \vec{\kappa}, \omega\right)
$$

where

$$
\begin{aligned}
& S\left(\overrightarrow{\mathrm{k}}_{2}-\overrightarrow{\mathrm{k}}_{1} ; \vec{\kappa}, \omega\right)= \sum_{\lambda, \lambda^{\prime}} P_{\lambda} \mid\left\langle\lambda^{\prime}\right| \sum_{j} \tilde{U}(\vec{\kappa}) e^{i \vec{\kappa} \cdot \overrightarrow{\mathrm{R}}_{j}} \\
&+\left.a \sum_{j} \tilde{U}\left(\overrightarrow{\mathrm{k}}_{2}-\overrightarrow{\mathrm{k}}_{1}+\vec{\kappa}\right) e^{i\left(\overrightarrow{\mathrm{k}}_{2}-\overrightarrow{\mathrm{k}}_{1}+\vec{\kappa}\right) \cdot \overrightarrow{\mathrm{R}}_{j}}|\lambda\rangle\right|^{2} \\
& \times \frac{1}{N} \delta\left(\hbar \omega+E_{\lambda}-E_{\lambda^{\prime}}\right) \\
& \tilde{U}(\vec{\kappa})=\int d \overrightarrow{\mathrm{r}} U(\overrightarrow{\mathrm{r}}) e^{i \vec{\kappa} \cdot \overrightarrow{\mathrm{r}}}
\end{aligned}
$$

and $\vec{\kappa}$ is the vector difference $\overrightarrow{\mathrm{k}}_{1}-\overrightarrow{\mathrm{k}}^{\prime}$. The $\delta$ function can be written in the form

$$
\begin{aligned}
& \delta\left(\hbar \omega-E_{\lambda}-E_{\lambda^{\prime}}\right) \\
& =\frac{1}{2 \pi \hbar} \int_{-\infty}^{\infty} d t \exp \left[-i t\left(\hbar \omega+E_{\lambda}-E_{\lambda^{\prime}}\right) / \hbar\right]
\end{aligned}
$$

The Heisenberg operator is given by

$$
\overrightarrow{\mathrm{R}}_{j}(t)=e^{i t H / \hbar} \overrightarrow{\mathrm{R}}_{j} e^{-i t H / \hbar},
$$

where $H$ is the Hamiltonian of the target nucleus. From Eqs. (2.8), (2.10), and (2.11), one gets

$$
\begin{aligned}
& S\left(\overrightarrow{\mathrm{k}}_{2}-\overrightarrow{\mathrm{k}}_{1} ; \vec{\kappa}, \omega\right)=\frac{1}{2 \pi \hbar N} \int_{-\infty}^{\infty} d t e^{-i \omega t} \sum_{\lambda} P_{\lambda} \sum_{i, j}\langle\lambda|\left[\tilde{U} *(\vec{\kappa}) e^{-i \vec{\kappa} \cdot \overrightarrow{\mathrm{R}}_{i}(0)}+a^{*} U^{*}\left(\overrightarrow{\mathrm{k}}_{2}-\overrightarrow{\mathrm{k}}_{1}+\vec{\kappa}\right) e^{-i\left(\overrightarrow{\mathrm{k}}_{2}-\overrightarrow{\mathrm{k}}_{1}+\vec{\kappa}\right) \cdot \overrightarrow{\mathrm{R}}_{i}(0)}\right] \\
& \times\left[\tilde{U}(\vec{\kappa}) e^{i \vec{\kappa} \cdot \overrightarrow{\mathrm{R}}_{j}(t)}+a \tilde{U}\left(\overrightarrow{\mathrm{k}}_{2}-\overrightarrow{\mathrm{k}}_{1}+\vec{\kappa}\right) e^{-i\left(\overrightarrow{\mathrm{k}}_{2}-\overrightarrow{\mathrm{k}}_{1}+\vec{\kappa}\right) \cdot R_{j}(t)}\right]|\lambda\rangle .
\end{aligned}
$$

In single-beam scattering, it is customary to introduce a pair-correlation function

$$
\begin{aligned}
G^{e}(\overrightarrow{\mathrm{r}}, t)= & \frac{1}{(2 \pi)^{3}} \int d \vec{\kappa} e^{-i \vec{\kappa} \cdot \overrightarrow{\mathrm{r}}} \frac{1}{N} \\
& \times \sum_{\lambda} P_{\lambda} \sum_{i, j}\left\langle\lambda\left|e^{-i \vec{\kappa} \cdot \overrightarrow{\mathrm{R}}_{i}(0)} e^{i \overrightarrow{\mathrm{k}} \cdot \overrightarrow{\mathrm{R}}_{j}(t)}\right| \lambda\right\rangle .
\end{aligned}
$$

In this expression, the two momentum transfers in the exponential are the same. The pair-correlation function plays an important role in the scattering of a single slow neutron beam, for it gives rise to a very simply and entirely general expression for the angular and energy distribution of scattering in the Born approximation. This function alone, however, is insufficient to describe the 
scattering of two coherent neutron beams. To overcome this, a pair-correlation function with unequal momentum transfers is introduced,

$$
\begin{aligned}
G\left(\vec{\kappa}_{1}, \vec{\kappa}_{2} ; \overrightarrow{\mathrm{r}}, t\right)= & \frac{1}{(2 \pi)^{3}} \int d \vec{\kappa} e^{-\vec{\kappa} \cdot \overrightarrow{\mathrm{r}}} \frac{1}{N} \\
& \times \sum_{\lambda} P_{\lambda} \sum_{i, j}\langle\lambda| e^{i\left(\vec{\kappa}_{1}-\vec{\kappa}\right) \cdot \overrightarrow{\mathrm{R}}_{i}(0)} \\
& \times e^{-i\left(\vec{\kappa}_{2}-\vec{\kappa}\right) \cdot \overrightarrow{\mathrm{R}}_{j}(t)}|\lambda\rangle,
\end{aligned}
$$

where $N$ is the total number of scattering nuclei. By using the convolution formula for the Fourier transform of an (order) product, one arrives at

$$
\begin{aligned}
G\left(\vec{\kappa}_{1}, \vec{\kappa}_{2} ; \overrightarrow{\mathrm{r}}, t\right)= & \frac{1}{N} \sum_{\lambda} P_{\lambda} \\
& \times \sum_{i, j} \int d \overrightarrow{\mathrm{r}}^{\prime} e^{i\left(\vec{\kappa}_{1}-\vec{\kappa}_{2}\right) \cdot \overrightarrow{\mathrm{r}}^{\prime}-i \vec{\kappa}_{1} \cdot \overrightarrow{\mathrm{r}}} \\
& \times\left\langle\lambda\left|\delta\left(\overrightarrow{\mathrm{r}}^{\prime}-\overrightarrow{\mathrm{r}}-\overrightarrow{\mathrm{R}}_{i}(0)\right) \delta\left(\overrightarrow{\mathrm{r}}^{\prime}-\overrightarrow{\mathrm{R}}_{j}(t)\right)\right| \lambda\right\rangle .
\end{aligned}
$$

In terms of the function $G\left(\vec{\kappa}_{1}, \vec{\kappa}_{2} ; \overrightarrow{\mathbf{r}}, t\right)$, one can rewrite Eq. (2.10) as

$$
\begin{aligned}
S\left(\overrightarrow{\mathrm{k}}_{2}-\overrightarrow{\mathrm{k}}_{1} ; \vec{\kappa}, \omega\right) & =\frac{1}{2 \pi \hbar} \int_{-\infty}^{\infty} d t e^{-i \omega t} \int d \overrightarrow{\mathrm{r}} e^{i \vec{\kappa} \cdot \overrightarrow{\mathrm{r}}}[\tilde{U} *(\vec{\kappa}) \tilde{U}(\vec{\kappa}) G(0,0 ; \overrightarrow{\mathrm{r}}, t) \\
& +|a|^{2} \tilde{U}^{*}\left(\overrightarrow{\mathrm{k}}_{2}-\overrightarrow{\mathrm{k}}_{1}+\vec{\kappa}\right) \tilde{U}\left(\overrightarrow{\mathrm{k}}_{2}-\overrightarrow{\mathrm{k}}_{1}+\vec{\kappa}\right) G\left(\overrightarrow{\mathrm{k}}_{1}-\overrightarrow{\mathrm{k}}_{2}, \overrightarrow{\mathrm{k}}_{1}-\overrightarrow{\mathrm{k}}_{2} ; \overrightarrow{\mathrm{r}}_{1} t\right) \\
& \left.+a^{*} \tilde{U^{*}}\left(\overrightarrow{\mathrm{k}}_{2}-\overrightarrow{\mathrm{k}}_{1}+\vec{\kappa}\right) \tilde{U}(\vec{\kappa}) G\left(\overrightarrow{\mathrm{k}}_{1}-\overrightarrow{\mathrm{k}}_{2}, 0 ; \overrightarrow{\mathrm{r}}, t\right)+a \tilde{U}^{*}(\overrightarrow{\mathrm{k}}) \tilde{U}\left(\overrightarrow{\mathrm{k}}_{2}-\overrightarrow{\mathrm{k}}_{1}+\vec{\kappa}\right) G\left(0, \overrightarrow{\mathrm{k}}_{1}-\overrightarrow{\mathrm{k}}_{2} ; \overrightarrow{\mathrm{r}}, t\right)\right] .
\end{aligned}
$$

Equation (2.16) states that the correlation function $G\left(\vec{\kappa}_{1}, \vec{\kappa}_{2} ; \vec{r}, t\right)$ is the proper function to use to analyze the angular and energy distributions of the scattered neutron in the scattering of two coherent neutron beams by general systems of nuclei, just as the correlation function $G^{e}(\overrightarrow{\mathrm{r}}, t)$ is the proper function with which to analyze the angular and energy distributions of a scattered neutron in the conventional single-beam neutron-scattering problem. Both correlation functions are related in that

$$
G(0,0 ; \overrightarrow{\mathrm{r}}, t)=G^{e}(\overrightarrow{\mathrm{r}}, t) \text {. }
$$

With the help of Eqs. (2.13) and (2.14), the above equation can be easily verified.

It is customary to introduce a microscope particle density operator

$$
D(\overrightarrow{\mathrm{r}}, t)=\sum_{j} \delta\left(\overrightarrow{\mathrm{r}}-\overrightarrow{\mathrm{R}}_{j}(t)\right)
$$

and to simplify the notion on the averaging over $\lambda$ by

$$
\langle\cdots\rangle=\sum_{\lambda} P_{\lambda}\langle\lambda|\cdots| \lambda\rangle .
$$

Thus,

$$
\begin{aligned}
G\left(\vec{\kappa}_{1}, \vec{\kappa}_{2} ; \overrightarrow{\mathrm{r}}, t\right) \\
=\frac{1}{N} \int d \overrightarrow{\mathrm{r}}^{\prime} e^{i\left(\overrightarrow{\mathrm{k}}_{1}-\vec{\kappa}_{2}\right) \cdot \overrightarrow{\mathrm{r}}^{\prime}-i \vec{\kappa}_{1} \cdot \overrightarrow{\mathrm{r}}} \\
\quad \times\left\langle D\left(\overrightarrow{\mathrm{r}}^{\prime}-\overrightarrow{\mathrm{r}}, 0\right) D\left(\overrightarrow{\mathrm{r}}^{\prime}, t\right)\right\rangle .
\end{aligned}
$$

In terms of the Fourier components of the density operator,

$$
\begin{aligned}
\tilde{D}(\overrightarrow{\mathrm{q}}, t) & =\int d \overrightarrow{\mathrm{r}} D(\overrightarrow{\mathrm{r}}, t) e^{-i \overrightarrow{\mathrm{q}} \cdot \overrightarrow{\mathrm{r}}} \\
& =\sum_{j} e^{-i \overrightarrow{\mathrm{q}} \cdot \overrightarrow{\mathrm{R}}_{j}(t)}
\end{aligned}
$$

the spatial transform of $G\left(\overrightarrow{\mathrm{K}}_{1}, \overrightarrow{\mathrm{K}}_{2} ; \overrightarrow{\mathrm{r}}, t\right)$ has the form

$$
\begin{aligned}
\tilde{G}\left(\vec{\kappa}_{1}, \vec{\kappa}_{2} ; \vec{\kappa}, t\right) & =\int d \overrightarrow{\mathrm{r}} e^{i \vec{\kappa} \cdot \overrightarrow{\mathrm{r}}} G\left(\vec{\kappa}_{1}, \vec{\kappa}_{2} ; \overrightarrow{\mathrm{r}}, t\right) \\
& =\frac{1}{N}\left\langle\tilde{D}\left(\vec{\kappa}-\vec{\kappa}_{1}, 0\right) \tilde{D}\left(-\vec{\kappa}+\vec{\kappa}_{2}, t\right)\right\rangle,
\end{aligned}
$$

and Eq. (2.16) can be rewritten

$$
\begin{aligned}
S\left(\overrightarrow{\mathrm{k}}_{2}-\overrightarrow{\mathrm{k}}_{1} ; \overrightarrow{\mathrm{k}}_{1}-\overrightarrow{\mathrm{k}}^{\prime}, \omega\right)= & \frac{1}{2 \pi \hbar N} \int_{-\infty}^{\infty} d t e^{-i \omega t}\left[\tilde{U}^{*}\left(\overrightarrow{\mathrm{k}}_{1}-\overrightarrow{\mathrm{k}}^{\prime}\right) \tilde{U}\left(\overrightarrow{\mathrm{k}}_{1}-\overrightarrow{\mathrm{k}}^{\prime}\right)\left\langle\tilde{D}\left(\overrightarrow{\mathrm{k}}_{1}-\overrightarrow{\mathrm{k}}^{\prime}, 0\right) \tilde{D}\left(\overrightarrow{\mathrm{k}}^{\prime}-\overrightarrow{\mathrm{k}}_{1}, t\right)\right\rangle\right. \\
& +|a|^{2} \tilde{U}^{*}\left(\overrightarrow{\mathrm{k}}_{2}-\overrightarrow{\mathrm{k}}^{\prime}\right) \tilde{U}\left(\overrightarrow{\mathrm{k}}_{2}-\overrightarrow{\mathrm{k}}^{\prime}\right)\left\langle\tilde{D}\left(\overrightarrow{\mathrm{k}}_{2}-\overrightarrow{\mathrm{k}}^{\prime}, 0\right) \tilde{D}\left(\overrightarrow{\mathrm{k}}^{\prime}-\overrightarrow{\mathrm{k}}_{2}, t\right)\right\rangle \\
& +a^{*} \tilde{U}^{*}\left(\overrightarrow{\mathrm{k}}_{2}-\overrightarrow{\mathrm{k}}^{\prime}\right) \tilde{U}\left(\overrightarrow{\mathrm{k}}_{1}-\overrightarrow{\mathrm{k}}^{\prime}\right)\left\langle\tilde{D}\left(\overrightarrow{\mathrm{k}}_{2}-\overrightarrow{\mathrm{k}}^{\prime}, 0\right) \tilde{D}\left(\overrightarrow{\mathrm{k}}^{\prime}-\overrightarrow{\mathrm{k}}_{1}, t\right)\right\rangle \\
& \left.+a \tilde{U} *\left(\overrightarrow{\mathrm{k}}_{1}-\overrightarrow{\mathrm{k}}^{\prime}\right) \tilde{U}\left(\overrightarrow{\mathrm{k}}_{2}-\overrightarrow{\mathrm{k}}^{\prime}\right)\left\langle\tilde{D}\left(\overrightarrow{\mathrm{k}}_{1}-\overrightarrow{\mathrm{k}}^{\prime}, 0\right) \tilde{D}\left(\overrightarrow{\mathrm{k}}^{\prime}-\overrightarrow{\mathrm{k}}_{2}, t\right)\right\rangle\right]
\end{aligned}
$$


The function $S\left(\vec{k}_{2}-\vec{k}_{1} ; \vec{k}_{1}-\vec{k}^{\prime}, \omega\right)$ will be referred to as the scattering law. For a single-beam scattering, the corresponding scattering law can be obtained from Eq. (2.23) by setting $a=0$,

$$
\begin{aligned}
S^{e}\left(\overrightarrow{\mathrm{k}}_{1}-\overrightarrow{\mathrm{k}}^{\prime}, \omega\right)= & \left.S\left(\overrightarrow{\mathrm{k}}_{2}-\overrightarrow{\mathrm{k}}_{1} ; \overrightarrow{\mathrm{k}}_{1}-\overrightarrow{\mathrm{k}}^{\prime}, \omega\right)\right|_{a=0} \\
= & \frac{1}{2 \pi \hbar N} \int_{-\infty}^{\infty} d t e^{-i \omega t} \\
& \times\left[\tilde{U}^{*}\left(\overrightarrow{\mathrm{k}}_{1}-\overrightarrow{\mathrm{k}}^{\prime}\right) \tilde{U}\left(\overrightarrow{\mathrm{k}}_{1}-\overrightarrow{\mathrm{k}}^{\prime}\right)\right. \\
& \left.\times\left\langle\tilde{D}\left(\overrightarrow{\mathrm{k}}_{1}-\overrightarrow{\mathrm{k}}^{\prime}, 0\right) \tilde{D}\left(\overrightarrow{\mathrm{k}}^{\prime}-\overrightarrow{\mathrm{k}}_{1}, t\right)\right\rangle\right] .
\end{aligned}
$$

From Eqs. (2.23) and (2.24), one obtains a simplified expression

$$
\begin{aligned}
S\left(\overrightarrow{\mathrm{k}}_{2}-\overrightarrow{\mathrm{k}}_{1} ; \overrightarrow{\mathrm{k}}_{1}-\overrightarrow{\mathrm{k}}^{\prime}, \omega\right)-S^{e}\left(\overrightarrow{\mathrm{k}}_{1}-\overrightarrow{\mathrm{k}}^{\prime}, \omega\right) & -|a|^{2} S^{e}\left(\overrightarrow{\mathrm{k}}^{2}-\overrightarrow{\mathrm{k}}^{\prime}, \omega\right) \\
= & \frac{1}{\pi \hbar N} \int_{-\infty}^{\infty} d t e^{-i \omega t} \operatorname{Re}\left[a \tilde{U}^{*}\left(\overrightarrow{\mathrm{k}}_{1}-\overrightarrow{\mathrm{k}}^{\prime}\right)\right. \\
& \left.\times \tilde{U}\left(\overrightarrow{\mathrm{k}}_{2}-\overrightarrow{\mathrm{k}}^{\prime}\right)\left\langle D\left(\overrightarrow{\mathrm{k}}_{1}-\overrightarrow{\mathrm{k}}^{\prime}, 0\right) \tilde{D}\left(\overrightarrow{\mathrm{k}}^{\prime}-\overrightarrow{\mathrm{k}}_{2}, t\right)\right\rangle\right] .
\end{aligned}
$$

The quantities on the left-hand side of the above equation are the experimentally measurable quantities. The expression on the right-hand side contains the phase information of the Born amplitude $\tilde{U}(\vec{\kappa})$. This quantity is not obtainable in singlebeam neutron-scattering experiments.

\section{HARMONIC POTENTIAL}

In Sec. II, a general theory of neutron scattering was studied. In further study, the motion and spatial distribution of the target nuclei will be specified. In this section a very simple case is considered in which the target is formed only by a single nucleus with mass $M$ and is bounded in an isotropic harmonic potential;

$$
H_{N}=\frac{1}{2 M} \overrightarrow{\mathrm{P}}^{2}+\frac{M}{2} \omega_{0}^{2} \overrightarrow{\mathrm{R}}^{2}
$$

where $\omega_{0}$ is the frequency of vibration. In the treatment of the harmonic oscillator problem, it is customary to introduce a set of new Bose operators

$$
\eta_{i}=\left(\frac{1}{2 M \hbar \omega_{0}}\right)^{1 / 2}\left(M \omega_{0} X_{i}+i P_{i}\right)
$$

and

$$
\eta_{i}^{\dagger}=\left(\frac{1}{2 M \hbar \omega_{0}}\right)^{1 / 2}\left(M w_{0} X_{i}-i P_{i}\right) \quad \text { for } i=1,2,3,
$$

where $X_{i}$ and $P_{i}$ are the rectangular coordinate components of the position vector $\vec{R}$ and momentum vector $\overrightarrow{\mathrm{P}}$. These operators satisfy the commutator relations

$$
\left[\eta_{i}, \eta_{j}\right]=\left[\eta_{i}^{\dagger}, \eta_{j}^{\dagger}\right]=0
$$

and

$$
\left[\eta_{i}, \eta_{j}^{\dagger}\right]=\delta_{i j}, \text { for } i, j=1,2,3 \text {. }
$$

From Eqs. (3.2) and (3.3), one can wite the Hamiltonian in Eq. (3.1) as

$$
H_{N}=\hbar \omega_{0}\left(\sum_{i=1}^{3} \eta_{i}^{\dagger} \eta_{i}+\frac{3}{2}\right)
$$

The eigenequation takes the form

$$
H_{N}\left|n_{1}, n_{2}, n_{3}\right\rangle=E\left(n_{1}, n_{2}, n_{3}\right)\left|n_{1}, n_{2}, n_{3}\right\rangle,
$$

with the eigenvalues

$$
E\left(n_{1}, n_{2}, n_{3}\right)=\hbar \omega_{0}\left(n_{1}+n_{2}+n_{3}+\frac{3}{2}\right),
$$

and eigenfunctions

$$
\begin{aligned}
& \left|n_{1}, n_{2}, n_{3}\right\rangle \\
& \quad=\left(n_{1} ! n_{2} ! n_{3} !\right)^{-1 / 2}\left(\eta_{1}^{\dagger}\right)^{n_{1}}\left(\eta_{2}^{\dagger}\right)^{n_{2}}\left(\eta_{3}^{\dagger}\right)^{n_{3}}|0\rangle .
\end{aligned}
$$

The eigenfunction $|0\rangle$ satisfies the condition

$$
\eta_{i}|0\rangle=0 \text {. }
$$

To calculate the coherent differential cross section, one has to evaluate the correlation function $\tilde{G}\left(\vec{\kappa}_{1}, \vec{\kappa}_{2} ; \vec{\kappa}, t\right)$ in Eq. (2.22), which in the present case has the form

$$
\begin{aligned}
\tilde{G}\left(\vec{\kappa}_{1}, \vec{\kappa}_{2} ; \vec{\kappa}, t\right)= & \sum_{n_{1}} \sum_{n_{2}} \sum_{n_{3}} P_{n_{1} n_{2} n_{3}} \\
& \times\left\langle n_{1}, n_{2}, n_{3}\right| e^{i\left(\vec{\kappa}_{1}-\vec{\kappa}\right) \cdot \overrightarrow{\mathrm{R}}(0)} \\
& \times e^{-i\left(\vec{\kappa}_{2}-\vec{\kappa}\right) \cdot \overrightarrow{\mathrm{R}}(t)}\left|n_{1}, n_{2}, n_{3}\right\rangle,
\end{aligned}
$$

with

$$
R(t)=\left(X_{1}(t), X_{2}(t), X_{3}(t)\right),
$$

and

$$
X_{i}(t)=\left(\frac{\hbar}{2 M \omega_{0}}\right)^{1 / 2}\left[\eta_{i} e^{-i \omega_{0} t}+\eta_{i}^{\dagger} e^{i \omega_{0} t}\right]
$$

for $i=1,2,3$.

The probability $P_{n_{1} n_{2} n_{3}}$ denotes the statistical weight on the thermal average over initial states of the oscillating nucleus, and has the Boltzmann value

$$
P_{n_{1} n_{2} n_{3}}=\frac{\exp \left[-\beta \hbar \omega_{0}\left(n_{1}+n_{2}+n_{3}+\frac{3}{2}\right)\right]}{\sum_{n_{1}} \sum_{n_{2}} \sum_{n_{3}} \exp \left[-\beta \hbar \omega_{0}\left(n_{1}+n_{2}+n_{3}+\frac{3}{2}\right)\right]},
$$

where the quantity $\beta^{-1}$ is equal to the temperature $T$, multiplied by the Boltzmann constant $k_{B}$. Because of the isotropic nature of the Hamiltonian, the quantum mechanical motion and the thermal 
distribution of each retangular component is independent. One may rewrite Eq. (3.10) as

$$
\begin{aligned}
\tilde{G}\left(\vec{\kappa}_{1}, \vec{\kappa}_{2} ; \vec{\kappa}, t\right)=\prod_{i=1}^{3}[ & \sum_{n_{i}} P_{n_{i}}\left\langle n_{i}\right| e^{i\left(\kappa_{1}-\kappa\right){ }_{i} X_{i}(0)} \\
& \left.\times e^{-i\left(\kappa_{2}-\kappa\right){ }_{i} X_{i}(t)}\left|n_{i}\right\rangle\right]
\end{aligned}
$$

where

$$
P_{n_{i}}=\frac{\exp \left[-\beta h \omega_{0}\left(n_{i}+\frac{1}{2}\right)\right]}{\sum_{n_{i}} \exp \left[-\beta \hbar \omega_{0}\left(n_{i}+\frac{1}{2}\right)\right]},
$$

and

$$
\left|n_{i}\right\rangle=\left(n_{i} !\right)^{-1 / 2}\left(\eta_{i}^{\dagger}\right)^{n_{i}}|0\rangle .
$$

To evaluate Eq. (3.14), one has to recall the iden- tity for the multiplication of exponential operators,

$$
e^{A} e^{B}=e^{A+B+C},
$$

with

$$
C=\frac{1}{2}[A, B]+\frac{1}{12}[[A, B], B]+\frac{1}{12}[[B, A], A]+\cdots,
$$

and Bloch's identity, ${ }^{3}$

$$
\sum_{n_{i}} P_{n_{i}}\left\langle n_{i}\left|e^{Q}\right| n_{i}\right\rangle=\exp \left(\frac{1}{2} \sum_{n_{i}} P_{n_{i}}\left\langle n_{i}\left|Q^{2}\right| n_{i}\right\rangle\right),
$$

where $Q$ is a linear function of the operators $\eta$ and $\eta^{\dagger}$. Upon using these identities, one arrives at

$$
\begin{aligned}
\tilde{G}\left(\vec{\kappa}_{1}, \vec{\kappa}_{2} ; \vec{\kappa}, t\right) & =\prod_{i=1}^{3} \exp \left\{\frac{1}{2}\left(\kappa_{1}-\kappa\right)_{i}\left(\kappa_{2}-\kappa\right)_{i}\left[X_{i}(0), X_{i}(t)\right]\right\} \exp \left(-\frac{1}{2} \sum_{n_{i}} P_{n_{i}}\left\langle n_{i}\left|\left[\left(\kappa_{1}-\kappa\right)_{i} X_{i}(0)-\left(\kappa_{2}-\kappa\right)_{i} X_{i}(t)\right]^{2}\right| n_{i}\right\rangle\right) \\
& =\prod_{i=1}^{3} \exp \left(-\frac{1}{2} \sum_{n_{i}} P_{n_{i}}\left\langle n_{i}\left|\left[\left(\kappa_{1}-\kappa\right)_{i} X_{i}(0)\right]^{2}+\left[\left(\kappa_{2}-\kappa\right)_{i} X_{i}(t)\right]^{2}-2\left(\kappa_{1}-\kappa\right)_{i}\left(\kappa_{2}-\kappa\right)_{i} X_{i}(0) X_{i}(t)\right| n_{i}\right\rangle\right) .
\end{aligned}
$$

With the help of Eqs. (3.4), (3.12), (3.15), and (3.16), one obtains the following identities:

$$
\begin{aligned}
& \sum_{n_{i}} P_{n_{i}}\left\langle n_{i}\left|\eta_{i}^{\dagger} \eta_{i}\right| n_{i}\right\rangle=\left(e^{\hbar \omega_{0} \beta}-1\right)^{-1}, \\
& \sum_{n_{i}} P_{n_{i}}\left\langle n_{i}\left|\left[X_{i}(0)\right]^{2}\right| n_{i}\right\rangle=\sum_{n_{i}} P_{n_{i}}\left\langle n_{i}\left|\left[X_{i}(t)\right]^{2}\right| n_{i}\right\rangle=\frac{\hbar}{2 M \omega_{0}}\left[1+2\left(e^{\hbar \omega_{0} \beta}-1\right)^{-1}\right],
\end{aligned}
$$

and

$$
\sum_{n_{i}} P_{n_{i}}\left\langle n_{i}\left|X_{i}(0) X_{i}(t)\right| n_{i}\right\rangle=\frac{\hbar}{2 M \omega_{0}}\left(e^{\hbar \omega_{0} \beta}-1\right)^{-1}\left(e^{-i \omega_{0} t}+e^{i \omega_{0} t+\hbar \omega_{0} \beta}\right) .
$$

By using these identities, Eq. (3.20) can be rewritten

$$
\begin{aligned}
G\left(\vec{\kappa}_{1}, \vec{\kappa}_{2} ; \vec{\kappa}, t\right) & =\exp \left(-\frac{\hbar}{4 M \omega_{0}}\left[\left(\vec{\kappa}_{1}-\vec{\kappa}\right)^{2}+\left(\vec{\kappa}_{2}-\vec{\kappa}\right)^{2}\right] \operatorname{coth}\left(\frac{1}{2} \hbar \omega_{0} \beta\right)\right) \\
& \times \exp \left(\frac{\hbar}{M \omega_{0}}\left(\vec{\kappa}_{1}-\vec{\kappa}\right) \cdot\left(\vec{\kappa}_{2}-\vec{\kappa}\right) \operatorname{csch}\left(\frac{1}{2} \hbar \omega_{0} \beta\right) \cosh \left(\frac{1}{2} \hbar \omega_{0} \beta+i \omega_{0} t\right)\right) .
\end{aligned}
$$

\section{SCATTERING BY CRYSTAL}

The scattering of two coherent beams by an ideal static crystal was discussed in a previous paper. ${ }^{2}$ It was shown that through such a scattering, one can measure directly the phase of the crystal structure factor, which is not obtainable in a conventional single-beam scattering. Thermal vibrations of the crystal lattice were neglected in the study, and the energy exchange between the incident beam and the lattice system was completely ignored. For the scattering of electrons and protons by crystals, the neglect of these phenomena may be justified, since the energies of these quanta are, in general, much higher than those of the thermally excited vibrational quanta of the crystal, and the energy exchange between them is completely negligible. For particles as heavy as neutrons, however, properties of the system must be considered. The energies of neutrons with wavelengths suitable for interference scatterings are quite comparable with those of the vibrational 
quanta. It is known that in the crystal scattering of a single-neutron beam, the absorption or emission of these vibrational quanta causes a modification of the scattering amplitude. One expects a similar modification in the crystal scattering of two coherent neutron beams. The latter modification is the subject of the present study.

The target nuclei form a crystal. The equilibrium position of a nucleus is described by the lattice vector $\vec{r}_{i}$,

$$
\overrightarrow{\mathbf{r}}_{i}=\alpha_{i} \overrightarrow{\mathrm{a}}+\beta_{i} \overrightarrow{\mathrm{b}}+\gamma_{i} \overrightarrow{\mathrm{c}}, \text { for } i=1,2, \ldots, N,
$$

where $\vec{a}, \vec{b}$, and $\vec{c}$ are the basic vectors of the crystal. The parameters $\alpha_{i}, \beta_{i}$, and $\gamma_{i}$ are arbitrary integers. In the vibration, the position of the nucleus is given by

$$
\overrightarrow{\mathrm{R}}_{i}(t)=\overrightarrow{\mathrm{r}}_{i}+\overrightarrow{\mathrm{X}}\left(\overrightarrow{\mathrm{r}}_{i}, t\right),
$$

where $\vec{X}\left(\vec{r}_{i}, t\right)$ is the displacement vector. The vibration is described in terms of the harmonic approximation; hence, the Hamiltonian $H_{F}$ of the crystal is quadratic in terms of the displacement vectors. Thus,

$$
\begin{aligned}
H_{F}= & \sum_{i} \frac{1}{2 M} \overrightarrow{\mathrm{p}}^{2}\left(\overrightarrow{\mathrm{r}}_{i}, t\right)+\frac{1}{2} \sum_{i, j} \sum_{\alpha=1}^{3} \\
& \times \sum_{\beta=1}^{3} X_{\alpha}\left(\overrightarrow{\mathrm{r}}_{i}, t\right) A_{\alpha \beta}\left(\overrightarrow{\mathrm{r}}_{i}, \overrightarrow{\mathrm{r}}_{j}\right) X_{\beta}\left(\overrightarrow{\mathrm{r}}_{j}, t\right),
\end{aligned}
$$

where $A_{\alpha \beta}\left(\overrightarrow{\mathrm{r}}_{i}, \overrightarrow{\mathrm{r}}_{j}\right)$ is the second-order derivative of the potential energy of a nucleus in the crystal. It can be shown that the quantity $A_{\alpha \beta}\left(\overrightarrow{\mathrm{r}}_{i}, \overrightarrow{\mathrm{r}}_{j}\right)$ has the following properties:

$$
\begin{aligned}
A_{\alpha \beta}\left(\overrightarrow{\mathrm{r}}_{i}, \overrightarrow{\mathrm{r}}_{j}\right) & =A_{\alpha \beta}\left(\overrightarrow{\mathrm{r}}_{i}-\overrightarrow{\mathrm{r}}_{j}\right) \\
& =A_{\alpha \beta}\left(\overrightarrow{\mathrm{r}}_{j}-\overrightarrow{\mathrm{r}}_{i}\right)=A_{\beta \alpha}\left(\overrightarrow{\mathrm{r}}_{i}-\overrightarrow{\mathrm{r}}_{j}\right)
\end{aligned}
$$

and

$$
\sum_{j .} A_{\alpha \beta}\left(\overrightarrow{\mathrm{r}}_{i}-\overrightarrow{\mathrm{r}}_{j}\right)=0
$$

The normal modes of crystal vibrations are plane waves. For a wave of propagation vector $\vec{q}$, there are three normal modes with eigenfrequencies $\omega_{p}(\vec{q})(p=1,2,3)$ and their corresponding polarization eigenvectors $\vec{\sigma}^{p}(\vec{q})$. The eigenequation has the form

$$
\omega_{p}^{2}(\overrightarrow{\mathrm{q}}) \sigma_{\alpha}^{p}(\overrightarrow{\mathrm{q}})=\sum_{\beta} \tilde{A}_{\alpha \beta}(\overrightarrow{\mathrm{q}}) \cdot \sigma_{\beta}^{p}(\overrightarrow{\mathrm{q}})
$$

where

$$
\tilde{A}_{\alpha \beta}(\overrightarrow{\mathrm{q}})=\frac{1}{M} \sum_{1} A_{\alpha \beta}\left(\overrightarrow{\mathrm{r}}_{1}\right) e^{-i \overrightarrow{\mathrm{q}} \cdot \overrightarrow{\mathrm{r}}_{i}}
$$

is the dynamical matrix. It can be shown that the eigenvectors $\vec{\sigma} p(\vec{q})$ satisfy the following conditions:

$$
\begin{aligned}
& \sigma_{\alpha}^{* p}(\overrightarrow{\mathrm{q}})=\sigma_{\alpha}^{p}(-\overrightarrow{\mathrm{q}}), \\
& \sum_{p} \sigma_{\alpha}^{* p}(\overrightarrow{\mathrm{q}}) \sigma_{\beta}^{p}(\overrightarrow{\mathrm{q}})=\delta_{\alpha \beta},
\end{aligned}
$$

and

$$
\sum_{\alpha} \sigma_{\alpha}^{* p}(\overrightarrow{\mathrm{q}}) \sigma_{\alpha}^{p^{\prime}}(\overrightarrow{\mathrm{q}})=\delta_{p p^{\prime}}
$$

The lattice vibrations are quantized in the usual fashion. Displacement vectors and momenta are expanded in terms of creation and annihilation operators,

$$
\begin{aligned}
X_{\alpha}(\overrightarrow{\mathrm{r}}, t)= & \frac{V}{(2 \pi)^{3}} \int d \overrightarrow{\mathrm{q}} \sum_{p}\left(\frac{\hbar}{2 N M \omega_{p}(\overrightarrow{\mathrm{q}})}\right)^{1 / 2} \\
& \times\left\{\sigma_{\alpha}^{p}(\overrightarrow{\mathrm{q}}) \eta_{p}(\overrightarrow{\mathrm{q}}) \exp \left[i \overrightarrow{\mathrm{q}} \cdot \overrightarrow{\mathrm{r}}-i \omega_{p}(\overrightarrow{\mathrm{q}}) t\right]\right. \\
& \left.+\sigma_{\alpha}^{* p}(\overrightarrow{\mathrm{q}}) \eta_{p}^{\dagger}(\overrightarrow{\mathrm{q}}) \exp \left[-i \overrightarrow{\mathrm{q}} \cdot \overrightarrow{\mathrm{r}}+i \omega_{p}(\overrightarrow{\mathrm{q}}) t\right]\right\}
\end{aligned}
$$

and

$$
\begin{aligned}
P_{\alpha}(\overrightarrow{\mathrm{r}}, t)= & -i \frac{V}{(2 \pi)^{3}} \int d \overrightarrow{\mathrm{q}} \sum_{p}\left(\frac{\hbar M \omega_{p}(\overrightarrow{\mathrm{q}})}{2 N}\right)^{1 / 2} \\
& \times\left\{\sigma_{\alpha}^{p}(\overrightarrow{\mathrm{q}}) \eta_{p}(\overrightarrow{\mathrm{q}}) \exp \left[i \overrightarrow{\mathrm{q}} \cdot \overrightarrow{\mathrm{r}}-i \omega_{p}(\overrightarrow{\mathrm{q}}) t\right]\right. \\
& \left.-\sigma_{\alpha}^{* p}(\overrightarrow{\mathrm{q}}) \eta_{p}^{\dagger}(\overrightarrow{\mathrm{q}}) \exp \left[-i \overrightarrow{\mathrm{q}} \cdot \overrightarrow{\mathrm{r}}+i \omega_{p}(\overrightarrow{\mathrm{q}}) t\right]\right\},
\end{aligned}
$$

where $V$ is the volume of the crystal. The creation and annihilation operators commute with themselves, and

$$
\left[\eta_{p^{\prime}}(\overrightarrow{\mathrm{q}}), \eta_{p}^{\dagger}\left(\overrightarrow{\mathrm{q}}^{\prime}\right)\right]=\frac{(2 \pi)^{3}}{V} \delta_{p p^{\prime}} \delta\left(\overrightarrow{\mathrm{q}}-\overrightarrow{\mathrm{q}}^{\prime}\right) .
$$

In terms of these operators, the Hamiltonian $H_{F}$ in Eq. (4.3) reduces to the sum of $3 N$ independent harmonic oscillators,

$$
H_{F}=\frac{V}{(2 \pi)^{3}} \int d \overrightarrow{\mathrm{q}} \sum_{p} \hbar \omega_{p}(\overrightarrow{\mathrm{q}})\left[\eta_{p}^{\dagger}(\overrightarrow{\mathrm{q}}) \eta_{p}(\overrightarrow{\mathrm{q}})+\frac{1}{2}\right]
$$

Now, let us calculate the cross section for the scattering of two coherent neutron beams by nuclei undergoing harmonic vibrations on the lattice points of a crystal. In order to calculate the differential cross section in Eq. (2.7), one has to evaluate the correlation function, which according to Eq. (2.22) has the form

$$
\tilde{G}\left(\vec{\kappa}_{1}, \vec{\kappa}_{2} ; \vec{\kappa}, t\right)=\frac{1}{N}\left\langle\tilde{D}\left(\vec{\kappa}-\vec{\kappa}_{1}, 0\right) \tilde{D}\left(-\vec{\kappa}+\vec{\kappa}_{2}, t\right)\right\rangle,
$$

where the bracket $\langle\cdots\rangle$ stands for the thermal average of the expectation value with respect to the 
initial states of the lattice. It is assumed that the thermally excited quanta of the lattice forms a high-density Bose-Einstein gas. The thermal statistical weight $P_{\lambda}$ is the usual Boltzmann factor divided by the sum of states and has a form similar to the one given in Eq. (3.13). From Eqs. (2.21) and (4.2), one can rewrite E.q. (4.15) as

$$
\begin{aligned}
\tilde{G}\left(\vec{\kappa}_{1}, \vec{\kappa}_{2} ; \vec{\kappa}, t\right)= & \frac{1}{N} \sum_{i} \sum_{j} \exp \left[-i\left(\vec{\kappa}-\vec{\kappa}_{1}\right) \cdot \overrightarrow{\mathrm{r}}_{i}\right. \\
& \left.+i\left(\vec{\kappa}-\vec{\kappa}_{2}\right) \cdot \overrightarrow{\mathrm{r}}_{j}\right]\left\langle\exp \left[-i\left(\vec{\kappa}-\vec{\kappa}_{1}\right) \cdot \overrightarrow{\mathrm{X}}\left(\overrightarrow{\mathrm{r}}_{i}, 0\right)\right]\right. \\
& \left.\times \exp \left[+i\left(\vec{\kappa}-\vec{\kappa}_{2}\right) \cdot \overrightarrow{\mathrm{X}}\left(\overrightarrow{\mathrm{r}}_{j}, t\right)\right]\right\rangle .
\end{aligned}
$$

With the help of Eqs. (3.17), (3.18), and (3.19), the thermal expectation value in Eq. (4.1) becomes

$$
\begin{array}{r}
\tilde{G}\left(\vec{\kappa}_{1}, \vec{\kappa}_{2} ; \vec{\kappa}, t\right)=\frac{1}{N} \sum_{i} \sum_{j} \exp \left[-i\left(\vec{\kappa}-\vec{\kappa}_{1}\right) \cdot \overrightarrow{\mathrm{r}}_{i}+i\left(\vec{\kappa}-\vec{\kappa}_{2}\right) \cdot \overrightarrow{\mathrm{r}}_{j}\right] \exp \left\{\left\langle-\frac{1}{2}\left[\left(\vec{\kappa}-\overrightarrow{\kappa_{1}}\right) \cdot \overrightarrow{\mathrm{X}}\left(\overrightarrow{\mathrm{r}}_{i}, 0\right)\right]^{2}-\frac{1}{2}\left[\left(\vec{\kappa}-\vec{\kappa}_{2}\right) \cdot \overrightarrow{\mathrm{X}}\left(\overrightarrow{\mathrm{r}}_{j}, t\right)\right]^{2}\right.\right. \\
\left.\left.+\left[\left(\vec{\kappa}-\vec{\kappa}_{1}\right) \cdot \overrightarrow{\mathrm{X}}\left(\overrightarrow{\mathrm{r}}_{i}, 0\right)\right]\left[\left(\vec{\kappa}-\vec{\kappa}_{2}\right) \cdot \overrightarrow{\mathrm{X}}\left(\overrightarrow{\mathrm{r}}_{j}, t\right)\right]\right\rangle\right\} \cdot(4.17)
\end{array}
$$

In reaching Eq. (4.17), one has used the fact that the commutator $\left[\overrightarrow{\mathrm{X}}\left(\overrightarrow{\mathrm{r}}_{i}, 0\right), \overrightarrow{\mathrm{X}}\left(\overrightarrow{\mathrm{r}}_{j}, t\right)\right]$ is a $C$ number. From the expansion of $X_{\alpha}(\overrightarrow{\mathrm{r}}, t)$ in Eq. (4.11), it follows that

$$
\begin{aligned}
\left\langle X_{\alpha}\left(\overrightarrow{\mathrm{r}}_{i}, 0\right) X_{\beta}\left(\overrightarrow{\mathrm{r}}_{j}, t\right)\right\rangle= & \frac{\hbar}{2 N M^{\prime}} \frac{V^{2}}{(2 \pi)^{6}} \sum_{p} \sum_{p^{\prime}} \int d \overrightarrow{\mathrm{q}} d \overrightarrow{\mathrm{q}}^{\prime}\left[\omega_{p}(\overrightarrow{\mathrm{q}}) \omega_{p^{\prime}}(\overrightarrow{\mathrm{q}})\right]^{-1 / 2} \\
& \times\left\{\sigma_{\alpha}^{p}(\overrightarrow{\mathrm{q}}) \sigma_{\beta}^{* p^{\prime}}\left(\overrightarrow{\mathrm{q}}^{\prime}\right)\left\langle\eta_{p}(\overrightarrow{\mathrm{q}}) \eta_{p^{\prime}}^{\dagger}\left(\overrightarrow{\mathrm{q}}^{\prime}\right)\right\rangle \exp \left[i \overrightarrow{\mathrm{q}} \cdot \overrightarrow{\mathrm{r}}_{i}-\overrightarrow{\mathrm{q}}^{\prime} \cdot \overrightarrow{\mathrm{r}}_{j}+\omega_{p^{\prime}}\left(\overrightarrow{\mathrm{q}}^{\prime}\right) t\right]\right. \\
& \left.+\sigma_{\alpha}^{* p}(\overrightarrow{\mathrm{q}}) \sigma_{\beta}^{p^{\prime}}\left(\overrightarrow{\mathrm{q}}^{\prime}\right)\left\langle\eta_{p}^{\dagger}(\overrightarrow{\mathrm{q}}) \eta_{p^{\prime}}\left(\overrightarrow{\mathrm{q}}^{\prime}\right)\right\rangle \exp \left[-i \overrightarrow{\mathrm{q}} \cdot \overrightarrow{\mathrm{r}}_{i}+i \overrightarrow{\mathrm{q}}^{\prime} \cdot \overrightarrow{\mathrm{r}}_{j}-i \omega_{p^{\prime}}(\overrightarrow{\mathrm{q}}) t\right]\right\} .
\end{aligned}
$$

In Eq. (4.18), the vanishing thermal expectation values have been discarded,

$$
\left\langle\eta_{p}(\overrightarrow{\mathrm{q}}) \eta_{p^{\prime}}\left(\overrightarrow{\mathrm{q}}^{\prime}\right)\right\rangle=\left\langle\eta_{p}^{\dagger}(\overrightarrow{\mathrm{q}}) \eta_{p^{\prime}}^{\dagger}\left(\overrightarrow{\mathrm{q}}^{\prime}\right)\right\rangle=0 .
$$

Through the usual manner, as was done for Eq. (3.21), the nonvanishing thermal expectation values in Eq. (4.18) can be written

$$
\left\langle\eta_{p}(\overrightarrow{\mathrm{q}}) \eta_{p^{\prime}}^{\dagger}\left(\overrightarrow{\mathrm{q}}^{\prime}\right)\right\rangle=\frac{(2 \pi)^{3}}{V} \delta_{p p^{\prime}} \delta\left(\overrightarrow{\mathrm{q}}-\overrightarrow{\mathrm{q}}^{\prime}\right)\left[n_{p}(\overrightarrow{\mathrm{q}})+1\right]
$$

and

$$
\left\langle\eta_{p}^{\dagger}(\overrightarrow{\mathrm{q}}) \eta_{p^{\prime}}\left(\overrightarrow{\mathrm{q}}^{\prime}\right)\right\rangle=\frac{(2 \pi)^{3}}{V} \delta_{p p^{\prime}} \delta\left(\overrightarrow{\mathrm{q}}-\overrightarrow{\mathrm{q}}^{\prime}\right) n_{p}(\overrightarrow{\mathrm{q}}),
$$

where $n_{p}(\overrightarrow{\mathrm{q}})$ is the average quantum population

$$
n_{p}(\overrightarrow{\mathrm{q}})=\left(e^{\hbar B \omega_{p}(\vec{q})}-1\right)^{-1} \text {. }
$$

From Eqs. (4.20) and (4.21), a simpler form for Eq. (4.18) is obtained

$$
\begin{aligned}
\left\langle X_{\alpha}\left(\overrightarrow{\mathrm{r}}_{i}, 0\right) X_{\beta}\left(\overrightarrow{\mathrm{r}}_{j}, t\right)\right\rangle= & \frac{\hbar}{2 N M} \frac{V}{(2 \pi)^{3}} \sum_{p} \int d \overrightarrow{\mathrm{q}}\left[\omega_{p}(\overrightarrow{\mathrm{q}})\right]^{-1}\left\{\sigma_{\alpha}^{p}(\overrightarrow{\mathrm{q}}) \sigma_{\beta}^{* p}(\overrightarrow{\mathrm{q}})\left[n_{p}(\overrightarrow{\mathrm{q}})+1\right] \exp \left[i \overrightarrow{\mathrm{q}} \cdot\left(\overrightarrow{\mathrm{r}}_{i}-\overrightarrow{\mathrm{r}}_{j}\right)+i \omega_{p}(\overrightarrow{\mathrm{q}}) t\right]\right. \\
& \left.+\sigma_{\alpha}^{* p}(\overrightarrow{\mathrm{q}}) \sigma_{\beta}^{p}(\overrightarrow{\mathrm{q}}) n_{p}(\overrightarrow{\mathrm{q}}) \exp \left[-i \overrightarrow{\mathrm{q}} \cdot\left(\overrightarrow{\mathrm{r}}_{i}-\overrightarrow{\mathrm{r}}_{j}\right)-i \omega_{p}(\overrightarrow{\mathrm{q}}) t\right]\right\} .
\end{aligned}
$$

It is customary to expand the exponential in Eq. (4.17) containing the cross term of the displacement function as a power series. The first term gives the elastic contribution, and the second term gives the inelastic one-phonon contribution, etc. Thus,

$$
\begin{aligned}
\tilde{G}\left(\vec{\kappa}_{1}, \vec{\kappa}_{2} ; \vec{\kappa}, t\right)= & \frac{1}{N} \sum_{i} \sum_{j} \exp \left[-i\left(\vec{\kappa}-\vec{\kappa}_{1}\right) \cdot \overrightarrow{\mathrm{r}}_{i}+i\left(\vec{\kappa}-\vec{\kappa}_{2}\right) \cdot \overrightarrow{\mathrm{r}}_{j}\right] \exp \left[-W\left(\vec{\kappa}-\vec{\kappa}_{1}\right)-W\left(\vec{\kappa}-\vec{\kappa}_{2}\right)\right] \\
& \times\left\langle 1+\left[\left(\vec{\kappa}-\vec{\kappa}_{1}\right) \cdot \overrightarrow{\mathrm{X}}\left(\overrightarrow{\mathrm{r}}_{i}, 0\right)\right]\left[\left(\vec{\kappa}-\vec{\kappa}_{2}\right) \cdot \overrightarrow{\mathrm{X}}\left(\overrightarrow{\mathrm{r}}_{j}, 0\right)\right]+\cdots\right\rangle,
\end{aligned}
$$

where

$$
\begin{aligned}
W(\vec{\kappa}) & =\left\langle\frac{1}{2}\left[\vec{\kappa} \cdot \overrightarrow{\mathrm{X}}\left(\overrightarrow{\mathrm{r}}_{i}, 0\right)\right]^{2}\right\rangle \\
& =\frac{\hbar}{4 N M} \frac{V}{(2 \pi)^{3}} \sum_{p} \int d \overrightarrow{\mathrm{q}} \frac{\left|\overrightarrow{\mathrm{X}} \cdot \vec{\sigma}^{p}(\overrightarrow{\mathrm{q}})\right|^{2}}{\omega_{p}(\overrightarrow{\mathrm{q}})}\left[2 n_{p}(\overrightarrow{\mathrm{q}})+1\right],
\end{aligned}
$$


is the well-known Debye-Waller factor. ${ }^{9}$

From Eqs. (2.7), (2.22), (2.23), (4.23), and (4.24), one gets the elastic differential cross section,

$$
\begin{aligned}
\left(\frac{d^{2} \sigma}{d \Omega d E^{\prime}}\right)_{\mathrm{el}}=\frac{m^{2} k^{\prime}}{8 \pi^{3} \hbar^{5} k\left(1+|a|^{2}\right)} \int_{-\infty}^{\infty} d t e^{-i \omega t} \times & \mid \sum_{i}\left\{\tilde{U}\left(\overrightarrow{\mathrm{k}}_{1}-\overrightarrow{\mathrm{k}}^{\prime}\right) \exp \left[-W\left(\overrightarrow{\mathrm{k}}_{1}-\overrightarrow{\mathrm{k}}^{\prime}\right)+\left(\overrightarrow{\mathrm{k}}_{1}-\overrightarrow{\mathrm{k}}\right) \cdot \overrightarrow{\mathrm{r}}_{i}\right]\right. \\
& \left.+a \tilde{U}\left(\overrightarrow{\mathrm{k}}_{2}-\overrightarrow{\mathrm{k}}^{\prime}\right) \exp \left[-W\left(\overrightarrow{\mathrm{k}}_{2}-\overrightarrow{\mathrm{k}}^{\prime}\right)+i\left(\overrightarrow{\mathrm{k}}_{2}-\overrightarrow{\mathrm{k}}^{\prime}\right) \cdot \overrightarrow{\mathrm{r}}_{i}\right]\right\}\left.\right|^{2} .
\end{aligned}
$$

Carrying out the integration and eliminating the $\delta$ function, Eq. (4.26) can be rewritten

$$
\begin{aligned}
\left(\frac{d \sigma}{d \Omega}\right)_{\mathrm{el}}= & \frac{m^{2}}{4 \pi^{2} \hbar^{4}\left(1+|a|^{2}\right)} \mid \sum_{i}\left\{\tilde{U}\left(\overrightarrow{\mathrm{k}}_{1}-\overrightarrow{\mathrm{k}}^{\prime}\right) \exp \left[-W\left(\overrightarrow{\mathrm{k}}_{1}-\overrightarrow{\mathrm{k}}^{\prime}\right)+i\left(\overrightarrow{\mathrm{k}}_{1}-\overrightarrow{\mathrm{k}}^{\prime}\right) \cdot \overrightarrow{\mathrm{r}}_{i}\right]\right. \\
& \left.+a \tilde{U}\left(\overrightarrow{\mathrm{k}}_{2}-\overrightarrow{\mathrm{k}}^{\prime}\right) \exp \left[-W\left(\overrightarrow{\mathrm{k}}_{2}-\overrightarrow{\mathrm{k}}^{\prime}\right)+i\left(\overrightarrow{\mathrm{k}}_{2}-\overrightarrow{\mathrm{k}}^{\prime}\right) \cdot \overrightarrow{\mathrm{r}}_{i}\right]\right\}\left.\right|^{2} .
\end{aligned}
$$

Besides the Debye-Waller factors, the above result is simply the differential cross section for the elastic scattering of two coherent neutron beams by a static lattice in the Born approximation. The summation in Eq. (4.27) is characteristically dependent on the directional difference $\left(\overrightarrow{\mathrm{k}}_{1}-\overrightarrow{\mathrm{k}}_{2}\right)$ of two coherent neutron beams. If

$$
\overrightarrow{\mathrm{k}}_{1}-\overrightarrow{\mathrm{k}}_{2} \neq \overrightarrow{\mathrm{K}}
$$

where $\vec{K}$ is a reciprocal-lattice vector of the crystal, then the scattering from the two coherent neutron beams is incoherent and the resulting scattering would seem to come from two incoherent beams. This is certainly an uninteresting case. If, on the other hand,

$$
\overrightarrow{\mathrm{k}}_{1}-\overrightarrow{\mathrm{k}}_{2}=\overrightarrow{\mathrm{K}}
$$

then the scattering from two coherent neutron beams are coherent and the resultant differential cross section has the form

$$
\begin{aligned}
\left(\frac{d \sigma}{d \Omega}\right)_{\mathrm{el} .}= & \frac{m^{2}}{4 \pi^{2} \hbar^{4}\left(\mathbf{1}+|a|^{2}\right)}\left|\sum_{i} \exp \left[i\left(\overrightarrow{\mathrm{k}}_{1}-\overrightarrow{\mathrm{k}}^{\prime}\right) \cdot \overrightarrow{\mathrm{r}}_{i}\right]\right|^{2} \\
& \times \mid \tilde{U}\left(\overrightarrow{\mathrm{k}}_{1}-\overrightarrow{\mathrm{k}}^{\prime}\right) \exp \left[-W\left(\overrightarrow{\mathrm{k}}_{1}-\overrightarrow{\mathrm{k}}^{\prime}\right)\right] \\
& +\left.a \tilde{U}\left(\overrightarrow{\mathrm{k}}_{2}-\overrightarrow{\mathrm{k}}^{\prime}\right) \exp \left[-W\left(\overrightarrow{\mathrm{k}}_{2}-\overrightarrow{\mathrm{k}}^{\prime}\right)\right]\right|^{2}
\end{aligned}
$$

It is clear from Eq. (4.30) that in the crystal scattering of two coherent thermal neutron beams, the phase of the Born amplitude can be determined. One is unable to determine this phase in the conventional scattering of a single neutron beam. Equation (4.30) also shows that through the DebyeWaller factor, the scattered intensity decreases with the mean-square displacement of nuclei in the crystal.

From Eqs. (2.7), (2.22), (2.23), (4.23), and (4.24), one can get the one-phonon inelastic differential cross section. This cross section can be written as a sum of three distinct terms. First, two terms are inelastic differential cross sections of a single beam. The third term is a cross term, which will be referred to as the net differential cross section of two coherent beams, and has the form

$$
\begin{aligned}
\left(\frac{d^{2} \sigma}{d \Omega d E^{\prime}}\right)_{\text {inel }}^{n}=\frac{m^{2} k^{\prime}}{4 \pi^{3} \hbar^{5} k\left(1+|a|^{2}\right)} \int_{-\infty}^{\infty} d t e^{-i \omega t} \operatorname{Re}\left\{a \tilde{U}^{*}\left(\overrightarrow{\mathrm{k}}_{1}-\overrightarrow{\mathrm{k}}^{\prime}\right) \tilde{U}\left(\overrightarrow{\mathrm{k}}_{2}-\overrightarrow{\mathrm{k}}^{\prime}\right) \exp \left[-W\left(\overrightarrow{\mathrm{k}}_{1}-\overrightarrow{\mathrm{k}}^{\prime}\right)-W\left(\overrightarrow{\mathrm{k}}_{2}-\overrightarrow{\mathrm{k}}^{\prime}\right)\right]\right. \\
\\
\times \sum_{i} \sum_{i} \exp \left[-i\left(\overrightarrow{\mathrm{k}}_{1}-\overrightarrow{\mathrm{k}}^{\prime}\right) \cdot \overrightarrow{\mathrm{r}}_{i}+i\left(\overrightarrow{\mathrm{k}}_{2}-\overrightarrow{\mathrm{k}}^{\prime}\right) \cdot \overrightarrow{\mathrm{r}}_{j}\right] \\
\\
\left.\times\left\langle\left[\left(\overrightarrow{\mathrm{k}}_{1}-\overrightarrow{\mathrm{k}}^{\prime}\right) \cdot \overrightarrow{\mathrm{X}}\left(\overrightarrow{\mathrm{r}}_{i}, 0\right)\right]\left[\left(\overrightarrow{\mathrm{k}}_{2}-\overrightarrow{\mathrm{k}}^{\prime}\right) \cdot \overrightarrow{\mathrm{X}}\left(\overrightarrow{\mathrm{r}}_{j}, 0\right)\right]\right\rangle\right\} .
\end{aligned}
$$

By using Eq. (4.30), it is easy to verify that the net differential cross section in Eq. (4.31) vanishes for $\overrightarrow{\mathrm{k}}_{1}-\overrightarrow{\mathrm{k}}_{2} \neq \overrightarrow{\mathrm{K}}$. If $\overrightarrow{\mathrm{k}}_{1}-\overrightarrow{\mathrm{k}}_{2}=\overrightarrow{\mathrm{K}}$, one arrives at 


$$
\begin{aligned}
\left(\frac{d^{2} \sigma}{d \Omega d E^{\prime}}\right)_{\text {inel }}^{n}=\frac{m^{2} k^{\prime}}{4 \pi^{2} \hbar^{3} M k}\left(\frac{V}{(2 \pi)^{3} N}\right) \operatorname{Re}\left[a \tilde{U}^{*}\left(\overrightarrow{\mathrm{k}}_{1}-\overrightarrow{\mathrm{k}}^{\prime}\right) \tilde{U}\left(\overrightarrow{\mathrm{k}}_{2}-\overrightarrow{\mathrm{k}}^{\prime}\right) \exp \left[-W\left(\overrightarrow{\mathrm{k}}_{1}-\overrightarrow{\mathrm{k}}^{\prime}\right)-W\left(\overrightarrow{\mathrm{k}}_{2}-\overrightarrow{\mathrm{k}}^{\prime}\right)\right] \sum_{p} \int d \overrightarrow{\mathrm{q}}\left[\omega_{p}(\overrightarrow{\mathrm{q}})\right]^{-1}\right. \\
\times\left(\left(\overrightarrow{\mathrm{k}}_{1}-\overrightarrow{\mathrm{k}}^{\prime}\right) \cdot \vec{\sigma}^{p}(\overrightarrow{\mathrm{q}})\left(\overrightarrow{\mathrm{k}}_{2}-\overrightarrow{\mathrm{k}}^{\prime}\right) \cdot \vec{\sigma}^{* p}(\overrightarrow{\mathrm{q}})\left[n_{p}(\overrightarrow{\mathrm{q}})+1\right] \delta\left(\omega-\omega_{p}(\overrightarrow{\mathrm{q}})\right)\right. \\
\quad \times\left|\sum_{i} \exp \left[i\left(\overrightarrow{\mathrm{q}}-\overrightarrow{\mathrm{k}}_{1}+\overrightarrow{\mathrm{k}}^{\prime}\right) \cdot \overrightarrow{\mathrm{r}}_{i}\right]\right|^{2} \\
+\left(\overrightarrow{\mathrm{k}}_{1}-\overrightarrow{\mathrm{k}}^{\prime}\right) \cdot \vec{\sigma} *{ }^{p}\left(\overrightarrow{\mathrm{q}}_{\mathrm{q}}\right)\left(\overrightarrow{\mathrm{k}}_{2}-\overrightarrow{\mathrm{k}}^{\prime}\right) \cdot \vec{\sigma}^{p}(\overrightarrow{\mathrm{q}}) n_{p}(\overrightarrow{\mathrm{q}}) \delta\left(\omega+\omega_{p}(\overrightarrow{\mathrm{q}})\right) \\
\left.\left.\times\left|\sum_{i} \exp \left[i\left(\overrightarrow{\mathrm{q}}+\overrightarrow{\mathrm{k}}_{1}-\overrightarrow{\mathrm{k}}^{\prime}\right) \cdot \overrightarrow{\mathrm{r}}_{i}\right]\right|^{2}\right)\right]
\end{aligned}
$$

The expression on the right-hand side contains a term

$$
\tilde{U}^{*}\left(\overrightarrow{\mathrm{k}}_{1}-\overrightarrow{\mathrm{k}}^{\prime}\right) \tilde{U}\left(\overrightarrow{\mathrm{k}}_{2}-\overrightarrow{\mathrm{k}}^{\prime}\right),
$$

which carries the phase information of the Born amplitude. The expression also contains another term,

$$
\left(\overrightarrow{\mathrm{k}}_{1}-\overrightarrow{\mathrm{k}}^{\prime}\right) \cdot \vec{\sigma}^{p}(\overrightarrow{\mathrm{q}})\left(\overrightarrow{\mathrm{k}}_{2}-\overrightarrow{\mathrm{k}}^{\prime}\right) \cdot \vec{\sigma}^{* p}(\overrightarrow{\mathrm{q}}),
$$

which carries phase information of the polarization vector. In single-beam neutron scattering, one is not able to extract the latter information because in this scattering the experimentally observed quantities depend only on the term

$$
\left|\left(\overrightarrow{\mathrm{k}}_{1}-\overrightarrow{\mathrm{k}}^{\prime}\right) \cdot \vec{\sigma}^{p}(\overrightarrow{\mathrm{q}})\right|^{2} \text {. }
$$

Now it has been demonstrated that even in the presence of the lattice vibrations, one is still able to measure the phase of the scattering amplitude through the scattering of two coherent beams.

Note added in proof. Recently I have learned some interesting experimental activities on the crystal diffractions of two coherent beams. The experiments of two coherent $\mathrm{x}$ rays were reported by $\mathrm{V}$. Bonse and M. Hart [Appl. Phys. Lett. 6, 155 (1965); 1, 99 (1965); Z. Phys. 188, 154 (1966); 190, 455 (1966); 194,1 (1966)]. The recent experiments of two coherent neutron beams were reported by H. Rauch, W. Treimer, and V. Bonse [Phys. Lett . A 4 7, 369 (1974)] and by R. Colella, A. W. Overhauser, and S. A. Werner [Phys. Rev. Lett. 34, $1472(1975)]$.
${ }^{1}$ L. Marton, J. A. Simpson, and J. A. Suddeth, Rev. Sci. Instrum. 25, 1099 (1954).

${ }^{2}$ Ming Chiang Li, Phys. Rev. A 9,1635 (1974); 10, 781 (1974); 11, 1101 (1975).

${ }^{3}$ R. E. Peierls, Quantum Theory of Solids (Oxford, London, 1955); W. Marshall and S. W. Lovesey, Theory of Thermal Neutron Scattering (Oxford, London, 1971).

${ }^{4} \mathrm{~J}$. A. Simpson, Rev. Mod. Phys. 28, 254 (1956); D. Gabor, ibid. 28, 261 (1956).

${ }^{5}$ L. Marton, Phys. Rev. 85, 1057 (1952); L. Marton, J. A. Simpson, and J. A. Suddeth, ibid. 90,490 (1953); L. Marton, Science 118, 470 (1953); G. Mollenstedt and H. Duker, Z. Naturforsch. 8 , 79 (1.953); Naturwis- senchaften $\underline{42}, 41$ (1954); H. Duker, Z. Naturforsch. 10,256 (1955).

${ }^{6} \mathrm{H}$. Maier-Leibnitz and T. Springer, Z. Phys. 167, 386 (1962); F. J. Landkammer, ibid. 189, $113(1966)$; P. Korpiun, ibid. 195, 146 (1966).

${ }^{7}$ L. Van Hove, Phys. Rev. 95, 249 (1954); R. J. Glauber, ibid. 98, 1692 (1955).

${ }^{8}$ R. Engleman and P. Levi, J. Math. Phys. 4, 105 (1963); S. Sunakawa, S. Yamasaki and T. Nishigori, Prog. Theor. Phys. 37, 1051 (1967).

${ }^{9}$ P. Debye, Ann. Phys. (N.Y.) 43,49 (1914); H. J. Lipkin, ibid. 9 , 332 (1960). 